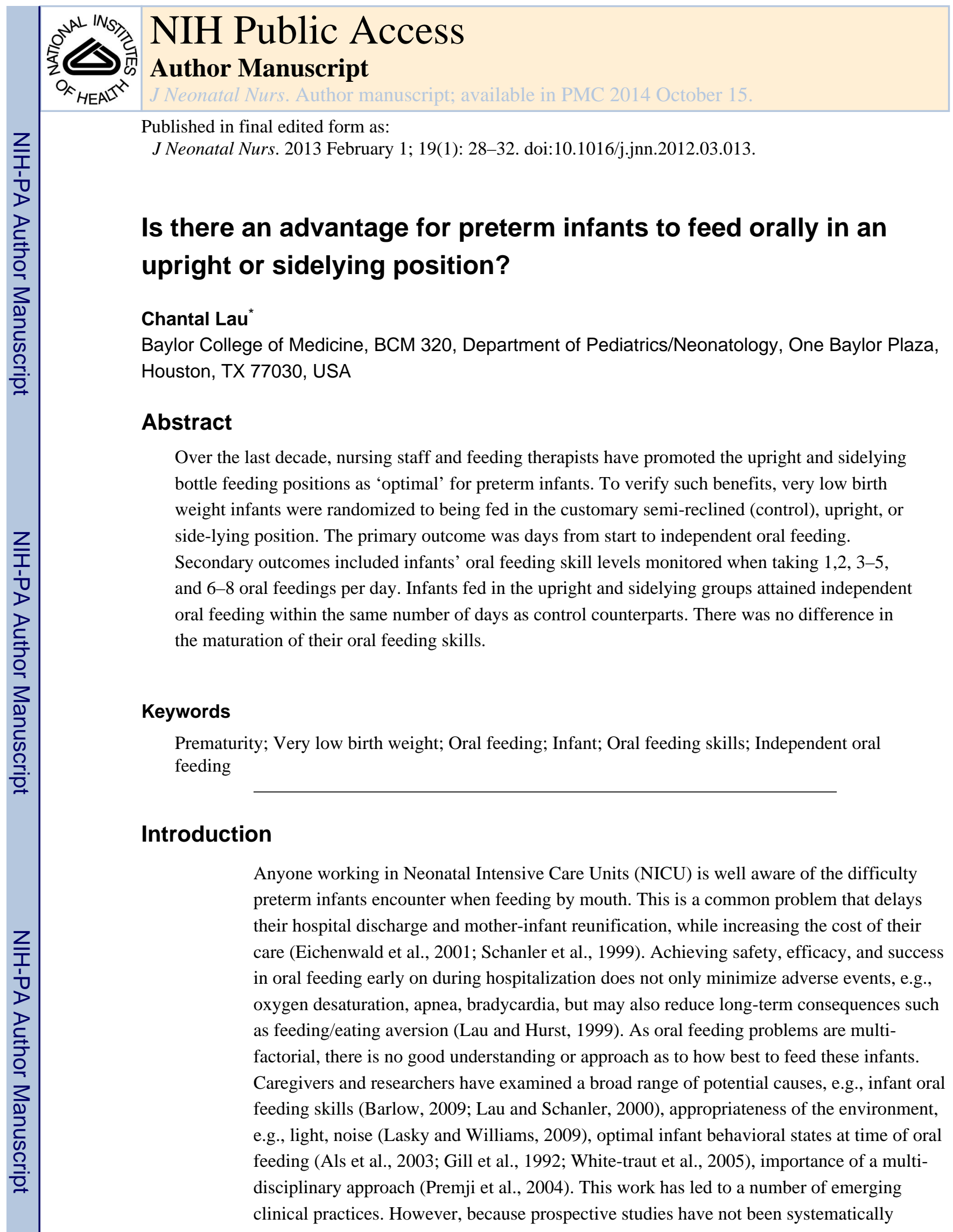

(C) 2012 Neonatal Nurses Association. Published by Elsevier Ltd. All rights reserved.

*Tel.: +1 832826 1384; fax: +1 832825 2799, clau@bcm.edu.. 
conducted prior to their introduction into the NICUs, evidence supporting a number of these practices lack evidence-based data to support their claimed benefits (Als et al., 2003; Beckman, 1986; Ludwig and Waitzman, 2007; Palmer et al., 1993; Thoyre et al., 2005). Consequently, health professionals regularly express reservation as to the true benefit(s) of specific approaches and/or interventions as any improvement may be simply the result of infants' maturation.

Among the many factors potentially impeding an infant's transition from tube to oral feeding, one frequently discussed by nursing staff and feeding therapists pertains to the 'optimal' feeding position an infant ought to be fed in. Over the last decade, caregivers have favored the upright over the customary semi-reclined feeding position, subsequently advocating the sidelying position. As no study to the author's knowledge has yet investigated the potential benefit(s) of bottle feeding infants in different positions, the present study was initiated. It was hypothesized that infants fed in either the upright or sidelying position will attain independent oral feeding faster than counterparts fed in the customary semi-reclined (control) position due to improved oral feeding skills.

\section{Study design/methods}

\section{Subjects}

Preterm infants (24.1-33.1 weeks gestation, GA) with birth weights ranging from 650 to $2085 \mathrm{~g}$ were recruited from the level II NICU at Texas Children's Hospital (Houston TX, USA). Exclusion criteria included gastrointestinal complications, congenital anomalies, or chronic medical conditions, e.g., intraventricular hemorrhage III and IV (Papile et al., 1978), periventricular leukomalacia, necrotizing enterocolitis, bronchopulmonary dysplasia. Only infants identified as 'feeders and growers' with a primary diagnosis of prematurity were enrolled. This diagnosis defined infants who were clinically stable, may have demonstrated clinical signs of immature systems, e.g., lung function, that resolved with maturation and whose hospital discharge was primarily based on their ability to feed by mouth; attainment of independent oral feeding being one of the discharge criteria recommended by the American Academy of Pediatrics (American Academy of Pediatrics - Policy Statement, 2008). This study was approved by the Baylor College of Medicine Institutional Review Board for Human Research. Parental consent was obtained following approval by attending neonatologists.

\section{Study design}

Infants were randomized to one of 3 study groups, i.e., upright, sidelying, and the semireclined control feeding position, with balanced GA and gender. Introduction and progression of oral feeding were left to the discretion of the attending physicians. All infants received the NICU standard of care which may include lactation consults, feeding therapists, and/or physical therapy as ordered. Only the feeding position of the infants differentiated them from each other. Duration and completion of oral feeding sessions followed the NICU protocol, i.e., they were left to the discretion of the individual caregivers based on their perception of infants' performance as a feeding progressed, e.g., disorganization, behavioral state, fatigue, apnea, bradycardia, oxygen desa-turation. Subjects were deemed to have 
attained independent oral feeding the first time they completed 8 oral feedings per day, 2 days in a row with no adverse events that did not self-resolve. That first successful day was defined as the day independent oral feeding was attained.

\section{Outcome measures}

Our primary outcome was days from start to independent oral feeding. Secondary outcomes included days of life (DOL), postmentrual age (PMA) at start of oral feeding (SOF) and independent oral feeding (IOF), and discharge. Additionally, infants' oral feeding skills (OFS) levels were monitored. OFS levels were identified based on infants respective proficiency (PRO; \% volume taken at $5 \mathrm{~min} /$ volume prescribed) and rate of milk transfer over the entire feeding (RT; $\mathrm{ml} / \mathrm{min}$ ) as described in an earlier study (Lau and Smith, 2011). Briefly, 4 OFS levels were defined: level 1, the most immature, with PRO $<30 \%$, RT $<1.5$ $\mathrm{ml} / \mathrm{min}$; level 2 with PRO $<30 \%$, RT $\geq 1.5 \mathrm{ml} / \mathrm{min}$; level 3 with PRO $\geq 30 \%$, RT $<1.5 \mathrm{ml} /$ $\mathrm{min}$, and level 4, the most mature, with PRO $\geq 30 \%$, RT $\geq 1.5 \mathrm{ml} / \mathrm{min}$. PRO, monitored during the first 5 min of a feeding, was used as an index most representative of infants' actual oral feeding skills as fatigue would be expected to be minimal. In contrast, RT, monitored during the entire feeding, was used as an index of endurance or fatigue as it reflected the resultant of their skills, fatigue, and/or occurrence of any adverse events. In taking into account infant's skills and endurance, this scale was used as an indicator of infants' feeding ability. As progression of daily oral feeding at Texas Children's Hospital is left to the discretion of the attending neonatologists, it was difficult to monitor these outcomes at fixed intervals of days as the rate of advancement varied between physicians. As such, OFS levels was measured when infants were taking 1,2, 3-5, and 6-8 oral feedings per day as this allowed us to capture at least 3 time periods during their transition from tube to independent oral feeding.

\section{Statistics}

Independent $t$-test assessed differences in the primary outcome and secondary outcomes between the individual experimental vs. control group. Fisher Exact test was used to compare the percent infants using a particular OFS level (1-4) at each monitored session between the individual experimental vs. control groups (WINPEPI; http:// www.brixtonhealth.com/pepi4windows.html). Significance was set at $p \leq 0.05$.

\section{Results}

Forty-one very low birth weight infants were recruited between 2008 and 2009. Their ethnic, racial, and gender distribution are presented in Table 1. Table 2 shows the similarity in subjects' characteristics between the experimental vs. control groups with the exception that infants in the sidelying group were of greater birth weight than their control counterparts ( $p$ $=0.036$ ). Table 3 describes the oral feeding progression of subjects from start to independent oral feeding (SOF-IOF). These outcomes were similar, but for the upright infants who were introduced to oral feeding at a younger PMA age than controls $(p=0.028)$. The primary outcome, number of days from SOF to IOF, between infants feeding in the upright, sidelying, and control positions was similar. Fig. 1 shows the OFS level profiles of infants when taking $1,2,3-5$, and $6-8$ oral feedings per day. For all 3 groups of infants, OFS 
level 1 declined over time as infants advanced in their oral feeding regimen while the most mature level, OFS 4, increased. OFS profiles were similar between the upright and sidelying vs. control group at all 3 time points.

\section{Discussion}

In general, clinical practices change over time gradually becoming 'standard of care'. This is usually based on medical advances providing evidenced-base support for or against a particular protocol. Recommendations provided by the American Academy of Pediatrics or meta-analyses conducted by organizations such as the Cochrane Reviews assist in such decision process.

As a lack of evidence-based data customarily raises skepticism from the part of health professionals, it is advantageous to confirm the benefit(s) of an approach or protocol prior to its implementation. Thus, the aim of this study was to determine whether the upright and/or sidelying feeding positions advocated by nursing staff and feeding therapists enhance infant oral feeding performance as measured by days from SOF to IOF and was associated with improved oral feeding skills.

The study design did not allow for blinding of group assignment. Nevertheless, despite caregivers favoring the 2 experimental feeding positions over that of the semi-reclined control, no difference was noted in the number of days from start to independent oral feeding for the 3 study groups. Although infants fed in the upright position were introduced to oral feeding at an earlier PMA than control counterparts and infants in the sidelying group had greater birth weights than controls, both groups attained independent oral feeding within the same number of days and at similar PMA. The additional observation that the profiles of infant OFS levels were also similar as the infants advanced in their oral feeding supports the notion that these 2 experimental feeding positions were not effective in enhancing oral feeding skills.

Caregivers' awareness has grown in regards to 4 broad categories of oral feeding deterrents: 1. infant medical condition, e.g., oxygen desaturation, apnea, bradycardia; 2 . behavioral state at time of feeding, e.g., the optimal state for oral feeding; 3. the NICU environment, e.g., light, sound, and 4. caregiver's approach to feeding. The establishment of developmental care programs primarily led by nursing staff focuses on minimizing their impact. Indeed, effort is made to cluster care or to carry out painful procedures within a specific time of the day rather than throughout the day. The light in nurseries is dimmed, isolette covers are used to reduce background light and noise, and containment methods (eg, swaddling) are used as calming interventions (Lau, 2006). However, it is well acknowledged that infants, even if born at the same gestational ages, do not necessarily present with the same shortcomings. This speaks for the importance of the individualized care concept advocated by Als et al. (1986). It is conceivable that benefit(s) of varying feeding positions may relate to the particular need(s) of some infants and not that of others. As such, it is suggested that proper characterization of the physiologic advantage(s) that such types of interventions may offer would first need to be identified before they can be used to their full potential. 
In summary, the hypothesis advanced in this study was not confirmed. The upright and sidelying feeding positions did not facilitate preterm infants' transition from tube to independent oral feeding. However, the observations presented herein do not necessarily negate the benefit(s) of these interventions, but rather raise the possibility that, although they do not have a broad-spectrum impact, they may assist infants with yet to be identified conditions.

\section{Acknowledgments}

The contents of this publication are solely the responsibility of the author and do not necessarily represent the official views of the National Institute of Child Health and Human Development or the National Institutes of Health. This project was supported by the National Institute of Child Health and Human Development (R01HD044469).

\section{Abbreviations}

$\begin{array}{ll}\text { DOL } & \text { days of life } \\ \text { GA } & \text { gestational age } \\ \text { IOF } & \text { independent oral feeding } \\ \text { NICU } & \text { Neonatal Intensive Care Unit } \\ \text { OFS } & \text { oral feeding skills } \\ \text { PMA } & \text { postmenstrual age } \\ \text { PRO } & \text { proficiency } \\ \text { RT } & \text { rate of transfer } \\ \text { SOF } & \text { start of oral feeding }\end{array}$

\section{References}

Als H, Lawhon G, Brown E, Gibes R, Duffy FH, Mcanulty G, Blickman JG. Individualized behavioral and environmental care for the very low birth weight preterm infant at high risk for bronchopulmonary dysplasia: neonatal intensive care unit and developmental outcome. Pediatrics. 1986; 78:1123-1132. [PubMed: 3786036]

Als H, Gilkerson L, Duffy FH, Mcanulty GB, Buehler DM, Vandenberg K, Sweet N, Sell E, Parad RB, Ringer SA, Butler SC, Blickman JG, Jones KJ. A three-center, randomized, controlled trial of individualized developmental care for very low birth weight preterm infants: medical, neurodevelopmental, parenting, and caregiving effects. J Dev Behav Pediatr. 2003; 24:399-408. [PubMed: 14671473]

American academy of pediatrics - policy statement. Hospital discharge of the high-risk neonate. Pediatrics. 2008; 122:1119-1126. [PubMed: 18977994]

Barlow SM. Central pattern generation involved in oral and respiratory control for feeding in the term infant. Curr Opin Otolaryngol Head Neck Surg. 2009; 17:187-193. [PubMed: 19417662]

Beckman, D. Oral Motor Assessment and Intervention. Beckman \& Assoc, Inc; 1986.

Eichenwald EC, Blackwell M, Lloyd JS, Tran T, Wilker RE, Richardson DK. Inter-neonatal intensive care unit variation in discharge timing: influence of apnea and feeding management. Pediatrics. 2001; 108:928-933. [PubMed: 11581446]

Gill NE, Behnke M, Conlon M, Anderson GC. Nonnutritive sucking modulates behavioral state for preterm infants before feeding. Scand J Caring Sci. 1992; 6:3-7. [PubMed: 1579769] 
Lasky RE, Williams AL. Noise and light exposures for extremely low birth weight newborns during their stay in the neonatal intensive care unit. Pediatrics. 2009; 123:540-546. [PubMed: 19171620]

Lau C, Hurst N. Oral feeding in infants. Curr Probl Pediatr. 1999; 29:105-124. [PubMed: 10202630]

Lau C, Schanler RJ. Oral feeding in premature infants: advantage of a self-paced milk flow. Acta Paediatr. 2000; 89:453-459. [PubMed: 10830459]

Lau C, Smith EO. A novel approach to assess oral feeding skills of preterm infants. Neonatology. 2011; 100:64-70. [PubMed: 21212698]

Lau C. Oral feeding in the preterm infant. Neoreviews. 2006; 7:e19-e27.

Ludwig SM, Waitzman KA. Changing feeding documentation to reflect infant-driven feeding practice. Newborn Infant Nurs Rev. 2007; 7:155-160.

Palmer MM, Crawley K, Blanco IA. Neonatal oral-motor assessment scale: a reliability study. J Perinatol. 1993; 13:28-35. [PubMed: 8445444]

Papile L, Burnstein J, Burnstein R, Kuffler H. Incidence and evolution of subependymal and intraventricular hemorrhage: a study of infants with birth weights less than 1500 grams. J Pediatr. 1978; 92:529-534. [PubMed: 305471]

Premji SS, Mcneil DA, Scotland J. Regional neonatal oral feeding protocol: changing the ethos of feeding preterm infants. J Perinat Neonatal Nurs. 2004; 18:371-384. [PubMed: 15646307]

Schanler RJ, Shulman RJ, Lau C, Smith EO, Heitkemper MM. Feeding strategies for premature infants: randomized trial of gastrointestinal priming and tube-feeding method [see comments]. Pediatrics. 1999; 103:434-439. [PubMed: 9925837]

Thoyre SM, Shaker CS, Pridham KF. The early feeding skills assessment for preterm infants. Neonatal Netw. 2005; 24:7-16. [PubMed: 15960007]

White-traut RC, Berbaum ML, Lessen B, Mcfarlin B, Cardenas L. Feeding readiness in preterm infants: the relationship between preterm behavioral state and feeding readiness behaviors and efficiency during transition from gavage to oral feeding. Mcn Am J Matern Child Nurs. 2005; 30:52-59. [PubMed: 15622150] 

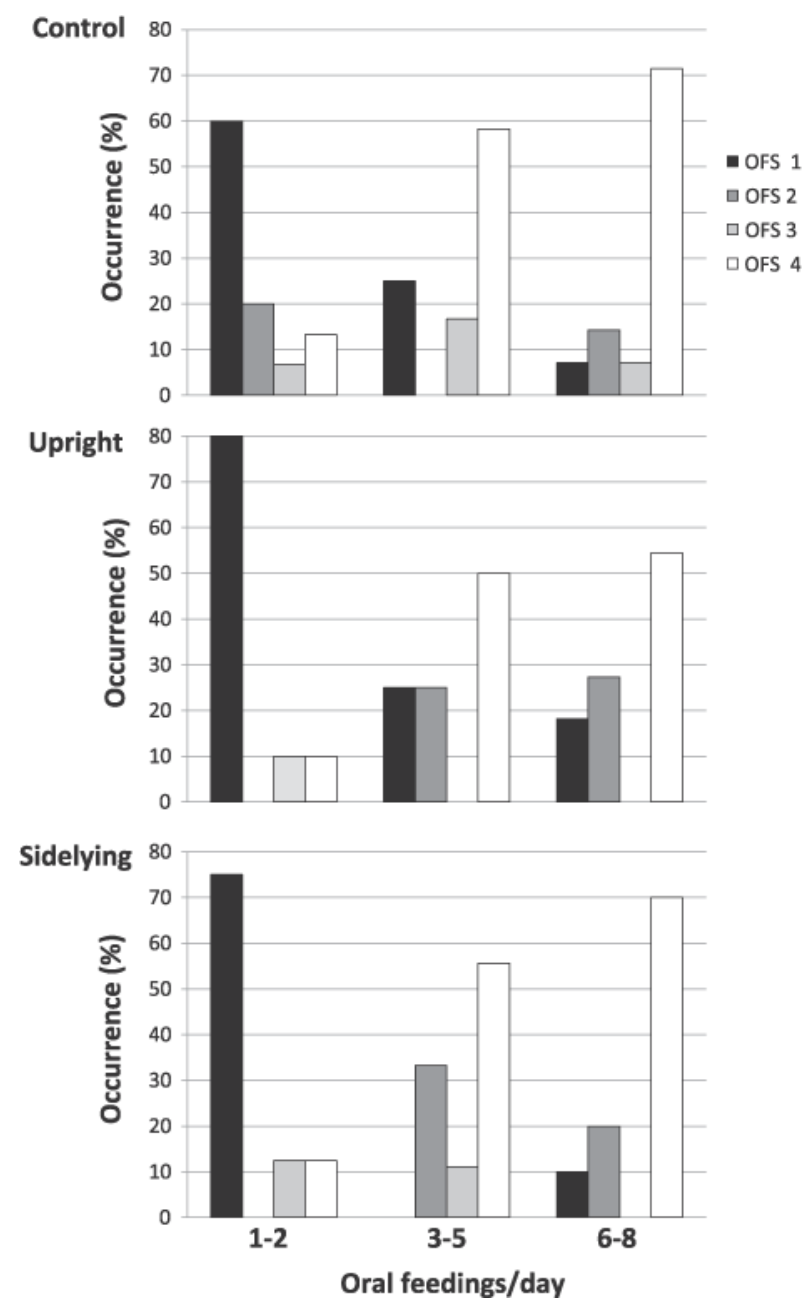

Fig. 1.

Oral feeding skill levels by group at $1,2,3-5$, and 6-8 oral feedings per day. 


\section{Table 1}

Subjects' ethnic/racial and gender distribution in the Upright and Sidelying studies.

\begin{tabular}{lrl}
\hline Ethnic/Racial & $\boldsymbol{N}$ & Percent \\
\hline Caucasian & 15 & 36.6 \\
Hispanic & 16 & 39.0 \\
African-American & 3 & 7.3 \\
Other ${ }^{2}$ & 7 & 17.1 \\
Gender & & \\
Male & 18 & 44 \\
Female & 23 & 56 \\
\hline aixed race/ethnicity. &
\end{tabular}




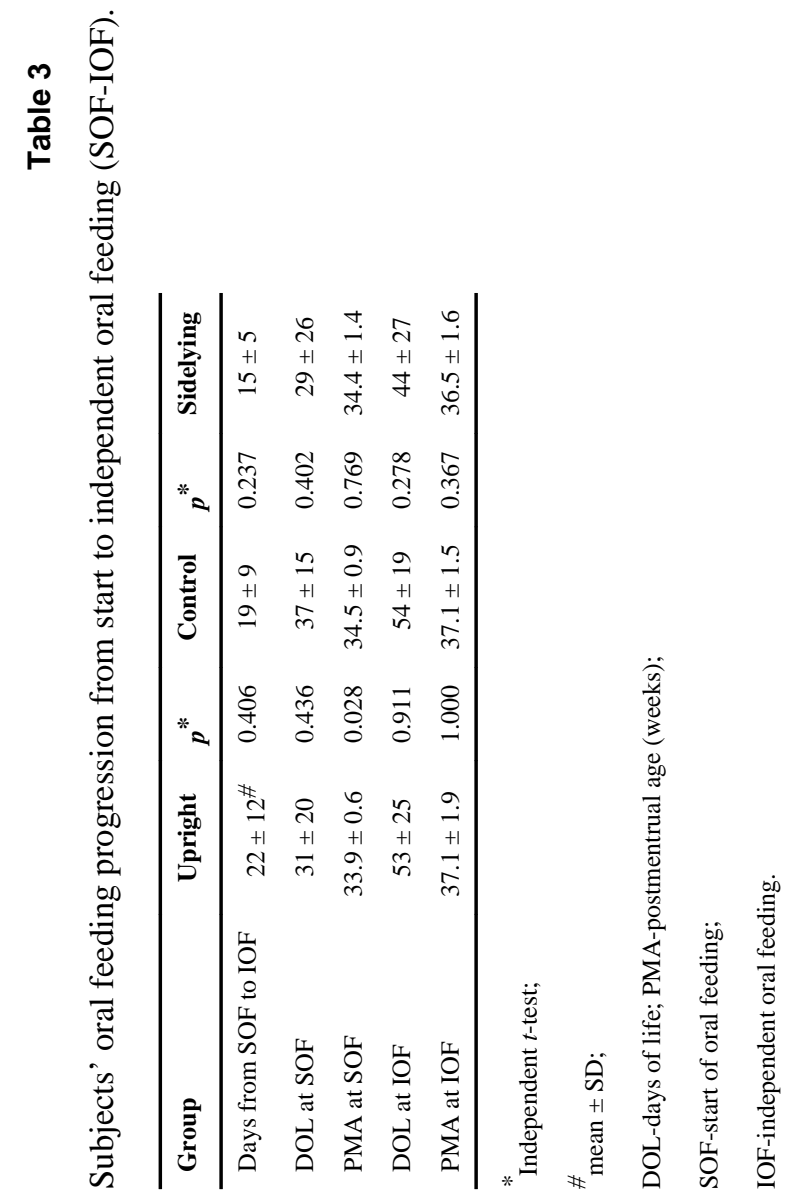

